\title{
The Development and Real-World Deployment of FROG, the Fun Robotic Outdoor Guide
}

\author{
Vanessa Evers ${ }^{1}$, Nuno Menezes ${ }^{2}$, Luis Merino ${ }^{3}$, Dariu Gavrila ${ }^{4}$, Fernando Nabais ${ }^{2}$, Maja Pantic ${ }^{5}$, \\ Paulo Alvito ${ }^{6}$, Daphne Karreman ${ }^{1}$ \\ University of Twente ${ }^{1}$ (Enschede, The Netherlands), YDreams ${ }^{2}$ (Lisbon, Portugal), Pablo de Olavide University ${ }^{3}$ \\ (Seville, Spain), University of Amsterdam ${ }^{4}$ (Amsterdam, The Netherlands), Imperial College London ${ }^{5}$ (London, UK), \\ IDMind $^{6}$ (Lisbon, Portugal) \\ v.evers@utwente.nl
}

\begin{abstract}
This video details the development of an intelligent outdoor Guide robot. The main objective is to deploy an innovative robotic guide which is not only able to show information, but to react to the affective states of the users, and to offer locationbased services using augmented reality. The scientific challenges concern autonomous outdoor navigation and localization, robust 24/7 operation, affective interaction with visitors through outdoor human and facial feature detection as well as engaging interactive behaviors in an ongoing non-verbal dialogue with the user.
\end{abstract}

\section{Categories and Subject Descriptors}

H.1.2 User/Machine Systems

\section{General Terms}

Design, Human Factors

\section{Keywords}

HRI, Guide Robots, Social Robots, Social Computing, HumanAware Navigation, Real world robot deployment, Affective Computing, Emotion Recognition, Multi-modal HRI

\section{INTRODUCTION}

The video describes the main objectives and challenges of project FROG. This project focuses on an emerging class of intelligent robot platforms that we call Outdoor Guide Robots. The proposed research will make autonomous outdoor robots into viable and significant location-based outdoor service providers with capacity to transform our experience of outdoor spaces, and, in particular, outdoor touristic scenarios.

The project aims to the development of an innovative robotic guide, which can enhance the experience of visitors of touristic places. While robotic guides have been developed in the past [3, 2], the FROG robot will generate an innovative installation that includes affective computing as the key to adapt to the users. We envision a robotic solution that can operate $24 / 7$ in outdoor environments, has an interesting personality that can engage small groups of visitors for part of their visit, it detects visitors groupformation and their engagement with the robot and it offers multiple interaction modalities and augmented reality content to

Permission to make digital or hard copies of part or all of this work for personal or classroom use is granted without fee provided that copies are not made or distributed for profit or commercial advantage and that copies bear this notice and the full citation on the first page. Copyrights for third-party components of this work must be honored. For all other uses, contact the Owner/Author. Copyright is held by the owner/author(s).

HRI'14, March 3-6 2014, Bielefeld, Germany

ACM 978-1-4503-2658-2/14/03.

http://dx.doi.org/10.1145/2559636.2559649 experience novel aspects of the site. This way, the robot will offer short enjoyable tours for families or friends that they can enjoy at their own pace while engaging with the content of the site.

The accompanying video submission shows the robots inner hardware and shell designs as well as tests that were run to assess user needs, autonomous mapping and localization and person tracking as well as engagement and affect detection. Next steps for the coming are the implementation of adaptive robot behaviors and content display through interaction with the visitors and in response to their engagement as measured by affect detection through facial feature extraction outdoors. The interaction modalities of the robots are currently audio, video display, movement and projection. These will be extended the next year with light and an antenna for gestural expression and pointing.

\section{GOALS AND CHALLENGES}

To achieve this goal, the project has to solve a series of challenging problems:

- Reliable autonomous outdoor robot operation.

- Automatic, multimodal vision-based recognition of spontaneous displays of conversational and affective signals.

- Localization and Navigation in populated and dynamic environments.

- Cognitive social-psychological evaluation of user responses to robots adaptive behaviors.

- Multi-modal human robot interaction and location based content.

A series of data collection, real-world technical deployment and user studies in the lab (for example: [1]) and in real world are carried out to guide the development of FROG at end-user sites (Royal Alcazar in Seville, Spain, and the Lisbon zoo in Portugal).

\section{ACKNOWLEDGEMENTS}

The research leading to these results received funding from the European Communitys 7th Framework programme under Grant agreement 288235. http://www.frogrobot.eu.

\section{REFERENCES}

[1] Karreman, D., et al. Picking favorites: The influence of robot eye-gaze on interactions with multiple users. In Proc. IROS (2013), 123-128

[2] Siegwart, R. et al. Robox at Expo.02: A large-scale installation of personal robots. Robotics and Autonomous Systems 42, 3-4 (March 2003), 203-222.

[3] Thrun, S. et al. Probabilistic algorithms and the interactive museum tour-guide robot minerva. The International Journal of Robotics Research 19 (October 2000), 972-999. 